\title{
Percepción de Actores Sociales sobre la Conducta Suicida: Análisis de Contenido a través de Grupos Focales ${ }^{4}$
}

\author{
Jorge Arturo Martínez Gómez \\ Dr. Psicología \\ Universidad Pedagógica y Tecnológica de Colombia \\ Tunja, Colombia \\ Correo electrónico: jorgearturo.martinez@uptc.edu.co
}

Adriana Cristina Robles Suárez

Psicóloga

Universidad Pedagógica y Tecnológica de Colombia

Tunja, Colombia

Correo electrónico: amadala18@hotmail.com
Recibido: 11/04/2016

Evaluado: 14/06/2016

Aceptado: 22/06/2016

Objetivo: determinar la percepción de los diferentes actores sociales sobre la conducta suicida en la ciudad de Valledupar (Cesar-Colombia). Método: Se abordó desde una perspectiva cualitativaexploratoria, con enfoque hermenéutico, mediante el uso de la técnica de grupos focales. Los participantes fueron 55 jóvenes y adultos (28 mujeres y 27 hombres), con edades comprendidas entre los 16 y 65 años, pertenecientes a la comunidad de Valledupar, los grupos estuvieron conformados por adolescentes, padres de familia, profesores y profesionales de la salud. Las categorías deductivas que se utilizaron como marco de referencia, partieron de las propuestas en el Modelo Ecológico del Desarrollo Humano de Bronfenbrenner. Resultados: se encontró que los participantes perciben que enfermedades mentales y físicas son predisponentes para que se pueda contemplar el suicidio, debido a que se encuentra en un estado vulnerable; demás, señalan que la edad, el género y la ausencia 0 presencia de hijos, son factores a la hora de contemplar la idea de quitarse la vida. Así mismo, se encontró que aspectos como la situación social y económica del país genera desesperanza frente al futuro, en especial en la población más joven, lo que se convierte en un estresor vital. Conclusión: el suicidio es un evento originado por múltiples variables de índole individual, social, familiar y cultural. Finalmente, se discute cómo estas variables deben ser incluidas en los planes de prevención e intervención de la política de Salud Mental.

Palabras clave

Conducta Suicida, Percepción, Factores Protectores, Factores de Riesgo, Salud Mental.

4 Para citar este artículo: Martínez, J., \& Robles, A. (2016). Percepción de actores sociales sobre la conducta suicida: Análisis de contenido a través de grupos focales. Informes Psicológicos, 16(2), pp. 53-68. http://dx.doi.org/10.18566/ infpsicv16n2a04 


\section{Perception of social actors on the suicidal be- havior: analysis of content through focus groups Abstract}

Objective: to determine the perception of the different social actors on suicidal behavior in the city of Valledupar (Cesar-Colombia). Method: it is addressed from a qualitative-exploratory perspective, with a hermeneutic approach, through the use of the focus groups technique. The participants were 55 youth and adults (28 women and 27 men), aged between 16 and 65 , belonging to the community of Valledupar. The groups were formed by adolescents, parents, teachers and health professionals. The deductive categories that were used as a frame of reference departed from the proposals of Bronfenbrenner's ecological framework for human development. Results: we found that participants perceive that mental and physical diseases are predisposing to contemplating suicide, since it is in a vulnerable state. In addition, they point out that the age, gender and the absence or presence of children are key factors when it comes to contemplating the idea of killing oneself. Likewise, it was found that aspects such as the social and economic situation of the country generates hopelessness about the future, especially in younger persons, which becomes a life stressor. Conclusion: suicide is an event caused by multiple variables of individual, social, family and cultural type. Finally, it is discussed how these variables should be included in the prevention and intervention plans of mental health policies.

Keywords suicidal behavior, perception, protective factors, risk factors, mental health.

\section{Percepção de atores sociais sobre a conduta suicida: análise de conteúdo através de grupos focais}

Resumo

Objetivo: determinar a percepção dos diferentes atores sociais sobre a conduta suicida na cidade de Valledupar (Cesar-Colômbia). Método: Se abordou desde una perspectiva qualitativa-exploratória, com enfoque hermenêutico, mediante 0 uso da técnica de grupos focais. Os participantes foram 55 jovens e adultos (28 mulheres e 27 homens), com idades compreendidas entre os 16 e 65 anos, pertencentes à comunidade de Valledupar, os grupos estiveram conformados por adolescentes, pais de família, professores e profissionais da saúde. As categorias dedutivas que se utilizaram como marco de referência, partiram das propostas no Modelo Ecológico do Desenvolvimento Humano de Bronfenbrenner. Resultados: se encontrou que os participantes percebem que doenças mentais e físicas são predisponentes para que se possa prever o suicídio, devido a que se encontra em um estado vulnerável, ademais, sinalam que a idade, o gênero e a ausência ou presença de filhos, são fatores na hora de prever a ideia de tira a vida. Assim mesmo, se encontrou que aspectos como a situação social e económica do país gera desesperança frente ao futuro, em especial na população mais jovem, o que se converte em um estressor vital. Conclusão: 0 suicídio é um evento originado por múltiplas variáveis de índole individual, social, familiar e cultural. Finalmente, se discute como esta variáveis devem ser incluídas nos planos de prevenção e intervenção da política de Saúde Mental.

Palavras chave conduta suicida, percepção, fatores protetores, fatores de risco, saúde mental. 


\section{ntroducción}

En los últimos años se ha observado un incremento de la conducta suicida, considerándose como un problema de Salud Pública y Mental, lo que genera preocupación a nivel mundial (Rocha, 2007). La Organización Mundial de la Salud (OMS, 2014) define la conducta suicida como toda acción empleada por una persona para causar daño a su integridad física y vital, se caracteriza por una serie de comportamientos sistemáticos que van desde la ideación en sus diferentes expresiones, pasando por las amenazas, los gestos e intentos, hasta el suicidio propiamente.

Según estadísticas de la OMS (2014), durante los últimos 50 años las tasas del suicidio se han incrementado en $60 \%$. Cada año se suicidan más de un millón de personas, lo que supone una tasa de mortalidad global de 16 por 100.000 habitantes o una muerte cada 40 segundos, además la misma organización calcula, que por cada muerte atribuible a esta causa se producen entre 10 y 20 intentos fallidos, lo que se traduce en lesiones, hospitalizaciones, traumas emocionales y mentales.

En los últimos años Colombia ha tenido un incremento en los índices de suicidio, lo que ubica en el tercer país Latinoamericano, después de Cuba y Brasil (Organización Mundial de la Salud, 2014).

El Instituto Nacional de Medicina Legal y Ciencia Forenses (INMLCF, 2014) en el informe sobre el Estudio del
Comportamiento Suicida en Colombia, señala que el suicidio es la cuarta forma de violencia en el país con una tasa de mortalidad para el año 2013 de 3.84 por cada 100.000 habitantes. Para el mes de noviembre de 2014, la misma entidad reportaba un total de 1453 suicidios.

Con respecto al género, según las estadísticas los hombres son los que más llevan a cabo el hecho de quitarse la vida. De los 1.810 suicidios registrados durante el año 2013, el 80.7\% corresponde a hombres con un total de 1.461 casos, y el 19.3\% a mujeres con un total de 349, es decir, por cada suicidio cometido por una mujer cuatro hombres lo realizan (Instituto Nacional de Medicina Legal, 2014).

Respecto a los factores de riesgo de la conducta suicida, las investigaciones señalan que es multicausal, por ejemplo, la OMS (2006) describe que el abuso de sustancias psicoactivas, la violencia recibida, las sensaciones de pérdida y diversos entornos culturales y sociales son factores de riesgo. Los resultados de investigaciones en los últimos años en Colombia, describen factores de riesgo psicosociales que varían dependiendo de las características socioculturales, asociadas al espectro suicida en jóvenes (ideación, planificación, intento y Suicidio). Estas variables son: problemas relacionados con el contexto escolar, la violencia intrafamiliar, el abuso sexual, el consumo de sustancias psicoactivas y bebidas alcohólicas, pertenecer a familias disfuncionales, presentar trastornos mentales y problemas económicos (Atehortúa et al., 2011; Vianchá, Bahamon, \& Alarcón, 2013). 
En una investigación desarrollada por Muñoz y Gutierres (2010) en Nariño (Colombia) encontraron que los principales factores predisponentes en el suicidio fueron los conflictos, la ausencia de una práctica religiosa, la carencia de una red de apoyo y la presencia de intentos previos de suicidio.

El INML (2013), en su informe sobre el comportamiento Suicida en Colombia, señala como factores de vulnerabilidad los celos (desconfianza e infidelidad) la enfermedad física o mental, las dificultades económicas, desamor, muerte de un familiar o un amigo, problemas escolares, educativas, jurídicos, laborales, conflictos familiares y suicidio de un familiar o amigo.

En cuanto a la percepción de la conducta suicida como fenómeno social, está sujeto a apreciaciones, construcciones y comprensiones derivadas de la manera en que diferentes factores psicosociales influyen en una persona o grupo social (Alvarez, Ojeda, \& Martínez, 2010). Por lo tanto, los estudios sobre percepción de suicidio se dirigen a describir e identificar los factores de riesgo y los factores protectores que las personas relacionan con la presencia o no de comportamientos suicidas (Álvarez et al., 2010; Villalobos, 2007).

Según Arlaes, Hernández y Álvarez (1998), una de las consecuencias a nivel social de la conducta suicida es el impacto emocional que causa en las personas que rodean a quien ejerce esta conducta, incrementando el riesgo de que ésta se presente nuevamente en otro de los miembros del grupo, porque se valida la conducta como una forma de resolución de problemáticas.
Es importante identificar factores que varían con la vulnerabilidad psicosocial en la población Colombiana; por esta razón, el objetivo de este trabajo de investigación fue determinar la percepción que tienen los diferentes actores sociales sobre la conducta suicida en la ciudad de Valledupar (Cesar-Colombia), para ser utilizado como sustrato en la elaboración de políticas públicas de salud mental, en la prevención e intervención del suicidio. La propuesta surge porque en los últimos tres años Valledupar ha presentado una tendencia creciente en el número de casos reportados de suicidios. Por ejemplo, el SIVIGILA reporta que en el 2012 se presentaron 19 casos, en el 2013 la cifra fue de 20 casos y para el 2014 el reporte fue de 24 casos. Esto sin contar con los casos fallidos que en el 2012 fueron de 133 y para el 2013, la cifra fue de 118 (SIVIGILA, 2013).

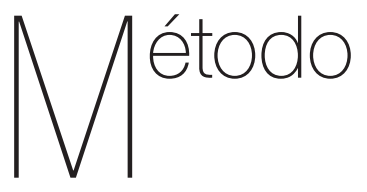

\section{Diseño}

Se utilizó un diseño de tipo exploratorio debido a que se buscaba hacer una aproximación inicial sobre la percepción de los participantes de la conducta suicida, para identificar factores de riesgo y protectores (Hernández, Fernández, \& Baptista, 2010).

La investigación se desarrolló desde el enfoque de la investigación cualitativa, definida por Bonilla y Rodríguez (1997) como el tipo de investigación que intenta hacer una aproximación global de 
las situaciones de las ciencias sociales para describirlas y comprenderlas de manera inductiva, es decir, a partir de los conocimientos, experiencias, creencias y representaciones que tienen las diferentes personas involucradas en ellas. Esto supone que los individuos interactúan con los otros miembros de su contexto social compartiendo el significado y el conocimiento que tienen de sí mismos y la realidad.

Igualmente, el enfoque de la investigación que se asumió fue el hermenéutico, donde la comprensión de la realidad social es susceptible de ser interpretada mediante el empleo de caminos metodológicos con particularidades muy propias (Hernández, Fernández, \& Baptista, 2010).

\section{Participantes}

La población fue constituida por los habitantes de la ciudad de Valledupar, Colombia, entre 15 y 65 años de edad $(M=33.9, D T=14)$. La muestra fue seleccionada por un muestreo por criterio lógico, que implica trabajar los casos que reúnan criterios predeterminados de importancia y en donde se tiene en cuenta la concurrencia en la repetición de la información o saturación como elemento básico de selección (Patton, 1990).

La muestra estuvo compuesta por 55 personas de las cuales 20 fueron adolescentes entre 15-17 años de edad, 10 padres de familia entre 45-50 años de edad, 15 fueron representantes de diferentes instituciones de la ciudad pertenecientes al Comité de Salud
Mental del municipio entre 28-45 años de edad, y los otros 10 fueron líderes comunales entre 35-50 años de edad.

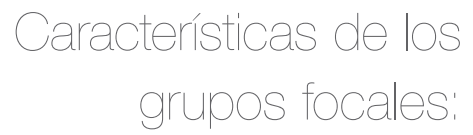

Primer grupo: Adolescentes hombres y mujeres, sin problemas graves de salud mental, que han tenido relación directa o indirecta con casos que han intentado o consumado el suicidio. Los adolescentes deben ser estudiantes de colegios de la ciudad de Valledupar, contar con el consentimiento informado firmado por los padres de familia y la institución académica y la manifestación de participación voluntaria a la investigación. Segundo grupo: Padre o madre de familia con hijos en la etapa adolescente, que han tenido relación directa o indirecta con casos que han intentado o consumado el suicidio. Grupo tres: Representantes de diferentes instituciones gubernamentales pertenecientes al Comité de Salud Mental, que estén trabajando en el diseño o la implementación de programas de prevención e intervención del suicidio en la política de Salud Mental de la ciudad de Valledupar. Cuarto grupo: líderes comunales donde se han presentado casos de intento o suicidios en su comunidad.

\section{Técnicas de recolección de información}

La técnica que se utilizó fueron los grupos focales, donde se generó un espacio de opinión para identificar los 
sentimientos y pensamientos de los individuos, buscando explicaciones de la conducta suicida para obtener datos cualitativos (Hernández, Fernández, \& Baptista, 2010).

\section{Procedimiento}

(a) Diseño de la guía de entrevista para los grupos focales; (b) Se hizo un acercamiento inicial a la población, se identificaron los participantes, se explicó el objetivo del trabajo y se extendió la invitación a participar voluntariamente en la investigación; (c) se recogieron los permisos correspondientes por parte de las instituciones educativas y consentimiento informado de los padres de familia y estudiantes; de igual manera, a las personas mayores se les explicó el consentimiento informado y posteriormente se firmó para su participación; (d) la guía de entrevistas para los grupos de enfoque fue sometida a una prueba piloto; (e) luego se fijaron los grupos focales, se recolectó la información en medio magnético y se transcribió al terminar las reuniones. Finalmente se realizó el análisis de los resultados obtenidos utilizando el sistema categorial o diseño sistemático propuesto por Sandoval, el cual permitió la construcción y validación de categorías para la estructuración del análisis de los datos cualitativos encontrados (Sandoval, 1996).

\section{Análisis de resultados}

Toda la información que surgió de los grupos focales, fue recopilada y agrupada en las categorías del modelo Ecológico (Microsistema, Mesosistema, Exosistema y Macrosistema) previamente establecidas como se puede observar en la Tabla 1.

El proceso contó primero con una fase de codificación descriptiva propia del primer contacto con los datos recolectados en las sesiones, luego se hizo una codificación axial, para hacer un proceso de conceptualización de los datos obtenidos; y finalmente se realiza la codificación selectiva para hacer una depuración empírica y conceptual, que incluye la triangulación y la contrastación para articular todo el sistema categorial construido a lo largo de la investigación.

En estos espacios destinados a la retroalimentación se discutieron los hallazgos más importantes y surgieron conclusiones que fueron soporte vital para la estructuración de la Política de Salud Mental.

Tabla 1.

Definición de categorías analíticas. Áreas correspondientes a los subsistemas del modelo ecológico

\begin{tabular}{ccc}
\hline $\begin{array}{c}\text { Categorías } \\
\text { deductivas }\end{array}$ & Operacionalización & Sub-categoría \\
\hline Microsistema & $\begin{array}{l}\text { Constituye el nivel más inmediato en el que se desa- } \\
\text { rrolla el individuo. El ambiente en el que el individuo } \\
\text { pasa más tiempo. (Bronfenbrenner, 1979). }\end{array}$ & $\begin{array}{l}\text { La percepción de los participantes sobre la manera } \\
\text { en que variables como el sexo, la edad, la historia mé- } \\
\text { dicar características propias de la personalidad, el } \\
\text { nivel educativo, las expectativas laborales, el manejo } \\
\text { del tiempo libre y las Pautas de Crianza influyen o no } \\
\text { en que se presente la conducta suicida. }\end{array}$ \\
\end{tabular}




\begin{tabular}{|c|c|c|}
\hline $\begin{array}{l}\text { Categorías } \\
\text { deductivas }\end{array}$ & Operacionalización & Sub-categoría \\
\hline Mesosistema & $\begin{array}{l}\text { El segundo nivel del modelo ecológico indaga el modo } \\
\text { en que las relaciones sociales cercanas por ejemplo, } \\
\text { con los amigos, con la pareja y con los miembros de } \\
\text { la familia aumentan el riesgo de convertirse en vícti- } \\
\text { ma o perpetradores de actos violentos. Incluye Fami- } \\
\text { lia, amigos, vida social (Bronfenbrenner, 1979). }\end{array}$ & $\begin{array}{l}\text { Percepción de los participantes sobre como las rela- } \\
\text { ciones con la familia, la situación económica familiar, } \\
\text { la relación con la pareja, las relaciones sociales, las } \\
\text { redes de apoyo aumentan o no el riesgo en presentar } \\
\text { comportamientos suicidas. }\end{array}$ \\
\hline Exosistema & $\begin{array}{l}\text { Es el tercer nivel. Se refiere a uno o más entornos } \\
\text { que no incluyen a la persona en desarrollo como } \\
\text { participante activo, pero en los cuales se producen } \\
\text { hechos que afectan a lo que ocurre en el entorno que } \\
\text { comprende a la persona en desarrollo, o que se ven } \\
\text { afectados por lo que ocurre en ese entorno. (Bronfen- } \\
\text { brenner, 1979). }\end{array}$ & $\begin{array}{l}\text { Percepción de los participantes sobre la manera en } \\
\text { que factores como el lugar y tipo de trabajo, el gru- } \\
\text { po de amigos que tiene la familia, la violencia en la } \\
\text { comunidad donde habita, la influencia de los medios } \\
\text { de comunicación con respecto a la conducta suicida } \\
\text { y las prácticas religiosas aumentan o no el riesgo de } \\
\text { presentar comportamientos suicidas. }\end{array}$ \\
\hline Macrosistema & $\begin{array}{l}\text { El cuarto y último nivel del modelo ecológico examina } \\
\text { los factores sociales más generales que determinan } \\
\text { las tasas de violencia. Se incluyen aquí los factores } \\
\text { que crean un clima de aceptación de la violencia, los } \\
\text { que reducen las inhibiciones contra esta, y los que } \\
\text { crean y mantienen las brechas entre distintos seg- } \\
\text { mentos de la sociedad, o generan tensiones entre di- } \\
\text { ferentes grupos o países. Se incluye el marco político, } \\
\text { normas culturales que apoyan la violencia como una } \\
\text { manera aceptable de resolver conflictos, actitudes } \\
\text { que consideran el suicidio como una opción personal } \\
\text { más que como un acto de violencia evitable (Bronfen- } \\
\text { brenner, 1979). }\end{array}$ & $\begin{array}{l}\text { Percepción de los participantes sobre la manera en } \\
\text { que factores como la Economía de la región, Políticas } \\
\text { de Salud Mental, el acceso a los servicios de Salud } \\
\text { y atención oportuna frente al evento del suicidio, así } \\
\text { como las creencias culturales sobre el suicidio y la } \\
\text { concepción sobre la Violencia, se vuelven factores de } \\
\text { riesgo o no y/o a la vez constituyen el evento como } \\
\text { una manera de resolver los problemas. }\end{array}$ \\
\hline
\end{tabular}

\section{Pesultados}

\section{Factores de riesgo de la conducta suicida en los sistemas del Modelo Ecológico}

En los diferentes grupos se plantearon factores de riesgos individuales, familiares y sociopolíticos, los cuales son percibidos como determinantes para que se presente la conducta suicida (Ver Tabla 2). Los grupos de discusión señalaron como dificultades para que las personas contemplen el suicidio, el presentar una enfermedad mental y física. Además, señalan categorías como la edad, el género y la ausencia o presencia de hijos, como factores que inciden a la hora de contemplar la idea de quitarse la vida. Así mismo, se encontró que los participantes perciben que las condiciones actuales tanto económicas y políticas del país generan incertidumbre frente al futuro, con mayor impacto en los más jóvenes, lo que es considerado como un estresor vital. 
Tabla 2.

Factores de Riesgo de la Conducta Suicida en los Sistemas del Modelo Ecológico

\begin{tabular}{|c|c|}
\hline Categoría & Factores de riesgo \\
\hline Microsistema & $\begin{array}{l}\text { Características personales del sujeto como: sexo, estado civil, presencia o ausencia hijos, nivel } \\
\text { educativo, decepciones amorosas o conflictos entre parejas, presencia de trastornos mentales, } \\
\text { historia médica, presencia de enfermedad física- biológica, intentos previos de suicidio, de- } \\
\text { presión, duelo no elaborado, consumo de sustancias psicoactivas, antecedentes de problemas } \\
\text { psicológicos, manejo inadecuado del tiempo libre, baja autoestima, dificultad para establecer } \\
\text { relaciones sociales, dificultades para resolver conflictos, déficit en las habilidades sociales }\end{array}$ \\
\hline Mesosistema & $\begin{array}{l}\text { Disfuncionalidad familiar, poca comunicación entre miembros de la familia, conflictos filio-pa- } \\
\text { rentales, padres estrictos, pautas de crianza inadecuadas, situación económica familiar, abuso } \\
\text { sexual, violencia intrafamiliar. }\end{array}$ \\
\hline \multirow{3}{*}{ Exosistema } & $\begin{array}{l}\text { Violencia de todo tipo como: sexual, género, comunidades, psicológica, pertenecer a una comu- } \\
\text { nidades LGTBI, intolerancia entre vecinos e intolerancia comunidad. }\end{array}$ \\
\hline & $\begin{array}{l}\text { Otros factores que influyen son: mitos y creencias sobre el suicidio, influencia negativa de me- } \\
\text { dios de comunicación, fácil acceso a métodos auto lesivos, mal manejo de la internet, poco con- } \\
\text { trol sobre páginas web, poco control sobre amistades negativas en redes sociales, poco apoyo } \\
\text { social y ayuda comunitaria, conflicto entre amigos, influencia negativa de amistades, intolerancia } \\
\text { hacia grupos diferenciales y ausencia de alguna práctica religiosa. }\end{array}$ \\
\hline & $\begin{array}{l}\text { Trabajo informal, pocas oportunidades laborales, desempleo, pobreza, situación económica y } \\
\text { social de la región. }\end{array}$ \\
\hline \multirow[t]{2}{*}{ Macrosistema } & $\begin{array}{l}\text { Dificultades para acceder a los servicios de salud, baja capacidad de respuesta en los servicios } \\
\text { de salud mental, deshumanización en los servicios de salud, desconocimiento de la normatividad } \\
\text { existente en salud mental, desconocimiento de los programas de salud mental. }\end{array}$ \\
\hline & $\begin{array}{l}\text { Situación social y político del país (guerras y conflictos bélicos que ha sufrido), desesperanza } \\
\text { frente al futuro, creencias sociales frente al suicidio y el acto de morir, creencias sobre el con- } \\
\text { sumo de sustancias psicoactivas. }\end{array}$ \\
\hline
\end{tabular}

Los grupos mencionaron la violencia en todas sus expresiones, los intentos previos de suicidio, la baja autoestima y las decepciones amorosas, como elementos que influye para que se presente la conducta suicida. Con respecto al sistema familiar, los factores que son percibidos como riesgo son la poca comunicación entre los miembros de la familia, las pautas de crianza inadecuadas y disfuncionalidad familiar.

\section{Factores de protectores de la conducta suicida en los sistemas del Modelo Ecológico}

Los grupos consideraron como principales factores protectores a nivel individual el tener un estilo de vida saludable, practicar algún deporte y en general tener buenos hábitos de salud (Ver Tabla 3). 
Tabla 3.

Factores protectores de la Conducta Suicida en los Sistemas del Modelo Ecológico

\begin{tabular}{|c|c|}
\hline Categoría & Factores de riesgo \\
\hline Microsistema & $\begin{array}{l}\text { Promover prácticas claves en salud mental; participar de actividades ludicas, estilos de vida } \\
\text { saludable procurar una buena alimentación, promover el autocuidado, mantener buena comuni- } \\
\text { cación, relación estable de pareja, solución positiva de conflictos, comportamientos asertivos, } \\
\text { conduct prosocial, tolerancia. }\end{array}$ \\
\hline Mesosistema & $\begin{array}{l}\text { Apoyo familiar, crear espacios para el diálogo fomentar adecuadas prácticas de crianza, fomen- } \\
\text { tar valores familiares adecuados, fomentar el respeto en la dinámica familiar, generar espacios } \\
\text { de diálogo para prevenir la violencia intrafamiliar y el maltrato, establecer normas de convivencia } \\
\text { basados en en el respeto y la tolerancia generar espacios familiares en donde se hable abierta- } \\
\text { mente de la conducta suicida y riesgos }\end{array}$ \\
\hline Exosistema & $\begin{array}{l}\text { Fortalecer tejido social, apoyo social, promover la convivencia ciudadana, promover el desarrollo } \\
\text { de las comunidades, generar espacios que incluyan poblaciónes vulnerables como poblacion } \\
\text { victima del conflicto y Igtbi, resignificar el valor de la vida, generar estrategias para rescatar } \\
\text { valores culturales, promover la educación basada en el respeto y la solidaridad, campañas de } \\
\text { prevencion del suicidio, educar a las comunidades sobre la importancia de promover la salud } \\
\text { mental, promover una educación basada en el respeto y las diferencias individuales, generar } \\
\text { mayor control sobre noticias relacionadas con el suicidio, utilizar redes sociales para prevenir } \\
\text { el suicidio, generar campañas de prevención del suicidio, redimensonar el concepto de salud } \\
\text { mental. }\end{array}$ \\
\hline Macrosistema & $\begin{array}{l}\text { Mejorar la calidad y la prestación de los servicios de salud, crear Polítcas Públicas de prevención } \\
\text { del Suicidio, redes nacionales contra el suicidio, Rescatar la identidad cultural del Pais, Ejercer } \\
\text { control sobre metodos autolesivos. }\end{array}$ \\
\hline
\end{tabular}

La comunidad también identificó que un aspecto clave para proteger la salud mental es resolver adecuadamente los conflictos, tener comportamientos asertivos y ser tolerante y respetuoso con las diferencias de los demás.

Los grupos consideraron como principales factores protectores al apoyo y contexto familiar, en las narrativas de los participantes se identificaron elementos como brindar apoyo al núcleo familiar, generar espacios para compartir con todos los miembros de la familia.

Con respecto al sistema social, la comunidad percibe que un factor importante que protege la salud mental de todos, son las buenas prácticas ciudadanas como la participación en los programas diseñados para la prevención del suicidio o en la participación libre y voluntaria en los procesos deliberativos que afecta el bien común de la comunidad, el acompañamiento de las entidades territoriales en programas de prevención y promoción de la salud mental en la comunidad. A nivel Macro, un factor protector importante es la consolidación de políticas públicas claras sobre prevención.

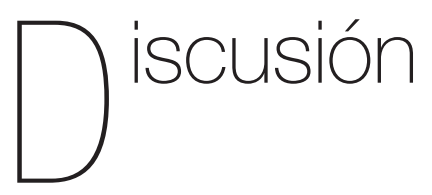

El objetivo de este trabajo fue determinar la percepción que tienen los diferentes actores sociales sobre la conducta suicida en la ciudad de Valledupar (Cesar-Colombia), para ser utilizado como sustrato en la elaboración de políticas 
públicas de salud mental, en la prevención e intervención del suicidio.

El punto de partida en el cual se apoya este trabajo es el fenómeno de la Percepción definida por Matlin y Foley (1996) como un fenómeno que incluye la interpretación de sensaciones dándoles resultado, significado y organización. Determinar la percepción de los actores sociales sobre la conducta suicida, poder reconocer y describir aquellos factores tanto de riesgo como protectores que rodean dicha conducta a partir de las experiencias de los diferentes actores sociales de la comunidad de Valledupar, condujo al análisis que permitió plantear nuevas bases para la Política Pública de Salud Mental que adelanta el municipio.

Llama la atención el hecho de que para la comunidad fue más fácil identificar los factores de riesgo que los factores protectores, confirmando los estudios de la OMS (2015) en donde se reporta que uno de los retos que tienen los gobiernos del mundo para prevenir el suicidio es precisamente educar y sensibilizar sobre aquellos factores que protegen la vida, puesto que estos son en su gran mayoría desconocidos por las comunidades; de esta manera se fortalece el tejido social y es más fácil prevenir el evento. Sin embargo, los programas que realizan prevención se centran en tres tipos de estrategias: (a) programas que incorporan psico-educación; (b) entrenamiento en detectar personas con riesgo y (c) pesquisa o screening para la detección de riesgo suicida. Además, no todos los programas cuentan con una evaluación sistemática de su efecto sobre el número de suicidios o bien sobre la conducta suicida, porque la mayoría de los programas no van dirigidos a la prevención directa del suicidio sino que su foco son factores asociados, como la depresión (Bustamante \& Florenzano, 2013). Por esto, una de las propuestas que se quiere implementar en la política para la prevención del suicidio en el municipio de Valledupar-Colombia, es la implementación de estrategias dirigidas al fortalecimiento de factores protectores en la comunidad.

Para la comprensión de los factores que influyen en la conducta suicida se utilizó el Modelo Ecológico de Bronfenbrenner, el cual estudia la conducta en cuatro sistemas. Se encontró que los participantes identifican el suicidio como un evento causado por multiples determinantes y factores sociales e individuales. Este resultado se ajusta a los encontrados por Ansari (2001, citado por LiamTam, 2011) el cual describió que existen muchos factores conjugados por los cuales una persona puede tomar la decisión de atentar contra su vida. Según el autor, las características personales del sujeto tales como la edad, la historia médica, o el consumo de drogas, unidos a alguna situación negativa por la que esté atravesando el sujeto, pueden confabularse y llevar a la persona a contemplar la idea de la muerte. De la misma manera; Mann (2014) consideran que el suicidio es un fenómeno biopsicosocial el cual se puede explicar como el resultado entre estresores vitales y predisposición o vulnerabilidad (Blandón, Quintero, García, \& Layne, 2015).

A nivel del Microsistema se encontró que los participantes perciben como factores de riesgo la historia médica del sujeto. Esta categoría incluye antecedentes de enfermedades físicas y trastornos mentales, las psicopatologías que más mencionaron fueron la Esquizofrenia y 
la depresión. De igual manera, esta categoría incluyó los intentos previos de suicidio y un duelo no elaborado. Estos hallazgos son similares a los resultados de algunos estudios; por ejemplo, Blandón y Andrade (2015) señalan que la conducta suicida suele verse incrementada a partir de los procesos psiquiátricos más o menos graves, en el 60\% de los casos de suicidio se observa de base un diagnóstico de enfermedad depresiva y en el $40 \%$ restante existen trastornos de personalidad, esquizofrenia, drogadicción y alcoholismo.

Con respecto a los intentos previos de suicidio, los participantes consideraron que para una persona que intente quitarse la vida, será más fácil que lo vuelva a intentar y tenga éxito, siendo esto un riesgo aún mayor. Sarmiento, Sánchez, Vargas y Álvarez (2010), igualmente encontraron que el $18.5 \%$ de las personas que tenían intentos fallidos de quitarse la vida, lo intentaban una segunda vez llegando a lograrlo.

Los conflictos entre parejas y la baja autoestima fueron identificados como factores de riesgo. Estas variables son atribuidas principalmente a la escasa habilidad para resolver los conflictos 0 carencia de habilidades sociales, que les permitan a las personas solventar las diferentes situaciones que plantea la vida cotidiana; lo anterior puede generar aislamiento social, y disfuncionalidad. Esta situación es vista como un estresor importante que puede conducir a algunos a contemplar la idea de suicidio. Esto se relaciona con lo descrito por Mansilla (2012), quien afirma que el déficit en habilidades sociales es una de las variables que más se ha encontrado en los perfiles de los suicidas españoles. De igual manera, Ojeda y Villalobos-Galvis (2011) encontraron que estresores vitales como las peleas frecuentes con la pareja, la baja autoestima, y en general la poca habilidad para resolver los conflictos, se convierten en uno de los principales factores que más guardan relación con los intentos de suicidio.

El grupo planteó como dificultades propias de los adolescentes las adicciones, mencionando el papel que desempeña el consumo de sustancias psicoactivas (incluido el alcohol) como factor de riesgo en la conducta suicida. Si bien las drogas son tenidas en cuenta como un factor de riesgo importante que puede predisponer al individuo a la conducta suicida, la percepción de los jóvenes frente al consumo de éstas y las consecuencias que puede traer esta conducta en la vida de las personas, se encontró dividida entre opiniones de tolerancia e incluso aceptación del consumo, hasta opiniones de rechazo hacia éste.

Para algunos jóvenes las drogas no se relacionan con la ideación suicida y menos con el hecho como tal, puesto que para ellos los efectos de las drogas son de relajación y estados de alegría. Mientras que otros jóvenes concluyeron que una persona que consume drogas y que a la vez esté pasando por un mal momento, tiene más posibilidades de atentar contra su vida puesto que no se tiene plena consciencia de los actos debido al efecto de la sustancia.

Estos resultados se muestran contradictorios a los expuestos por Jiménez, Carballo, Cano y Marín (2014) quienes afirman que el consumo de sustancias psicoactivas produce una pérdida del sentido de la realidad y, por lo tanto, 
dificultades el proceso de toma de decisiones que le permiten al individuo generar soluciones adaptativas, así como incrementa la probabilidad del uso de métodos más letales, lo cual aumenta también el riesgo y el acto suicida en sí mismo.

Por otra parte, las categorías del Mesosistema configuraron dos aspectos; la primera fue la relacionada con la dinámica familiar (incluye pautas de crianza, conflictos entre padres e hijos y violencia intrafamiliar). El grupo señala que la familia constituye un factor de riesgo y un factor protector; los participantes manifestaron que el no poder expresar las preocupaciones y los sentimientos de una manera abierta y confiada a los padres en el caso de los adolescentes, y a la pareja o hijos en el caso de los más adultos que participaron en las sesiones, puede ser negativo. Monge, Cubillas, Román y Abril (2007) encontraron que un ambiente familiar que no propicie las manifestaciones de afectividad y cariño, y en el que predomine la incomprensión y el rechazo, es un factor de riesgo relacionado con el intento de suicidio.

El segundo aspecto, se refiere a la escuela o colegio. Los participantes señalaron que algunas situaciones como el bullying, la exclusión y la discriminación, aumentan el riesgo del intento suicida. Si bien el Instituto Nacional de Medicina Legal no tiene datos exactos sobre la relación entre el suicidio y el matoneo, sí encontró que en el año 2013 al menos cinco casos de suicidio fueron vinculados directamente con el acoso escolar (INML, 2013).

Los participantes reportaron que en el colegio se presentan casos de violencia escolar debido a la orientación sexual, así como también burlas por el color de piel, limitaciones físicas y por el estrato socioeconómico. Estas situaciones sumadas al poco apoyo que los jóvenes encuentran en las directivas de los colegios, son percibidas como factores que pueden influir en la decisión de un joven a quitarse la vida. Frente a la violencia escolar como factor de riesgo para el suicidio, Keller, Sarchiapone y Zarrilli (2011), encontraron que cerca de un 30\% de los jóvenes que reportaron haber sido víctimas de violencia escolar también había intentado suicidarse debido al acoso. Así mismo, Borowsky (2013), encontró en un estudio llevado acabo con jóvenes escolarizados de Estados Unidos, que la mayoría de los casos de suicidios entre estudiantes de 6, 7 y 8 grado estuvieron relacionados con algún tipo de violencia verbal, física o psicológica por parte de sus compañeros.

Otros aspectos fundamentales fueron los hallados en el nivel del Exosistema. Una de las categorías que más llamó la atención fue la de los Medios de Comunicación. Esta categoría incluyó las redes sociales y los diferentes medios por donde se divulga la información. Si bien se reconoce que los medios de comunicación son una herramienta que puede ser muy útil para prevenir el evento, también perciben un problema en la manera en cómo son presentadas las notas relacionadas con el suicidio especialmente en algunos diarios de la ciudad, y este miedo se basa en el efecto de imitación de la conducta, especialmente en la población juvenil. Martínez, Moracén, Madrigal y Almanares, (1998) evidenciaron en un estudio que el $28 \%$ de la población evaluada que había intentado suicidarse, tenían amigos que se habían suicidado. 
La OMS (2015) advierte que los medios de comunicación tienen una labor importante en la prevención de la conducta suicida, sin embargo, señala que la información que dan los medios de comunicación sobre los eventos de suicidio en los países, deben ser regulada y tener un protocolo que permita dar un buen manejo de la información.

Otro aspecto que llamó la atención de los resultados fue lo relacionado con las prácticas religiosas. La percepción de los participantes frente a la creencia religiosa fue consistente todo el tiempo y se llegó a la conclusión de que creer o no creer en Dios es un factor de riesgo y un factor protector bastante significativo. El suicidio como conducta final, no es aceptado por mayoría de las religiones, quienes consideran el acto de quitarse la vida como una falta a las creencias y doctrinas de Dios. La función de la esfera espiritualidad como factor protector del suicidio se ha estudiado en los últimos años, donde aquellas personas que profesaban una religión son menos propensas a cometer el acto suicida independientemente que tuvieran algún tipo de ideación (Romero \& Gonnet, 2013). Estos resultados coinciden con lo expuesto por Taha, Florenzano, Sieverson, Aspillaga, y Alliende (2011), quienes señalan que la tasa de suicidio en aquellas personas que asistían semanalmente a oficios religiosos era del 11\%, en comparación con el $29 \%$ en aquellas que asistían con menor frecuencia.

Al indagar sobre sobre los factores de riesgo percibidos a nivel social y político (Macrosistema), se encontró que la percepción comunitaria frente al fenómeno del suicidio, se ve influenciada por variables como el desempleo, la situación social y el Sistema de Salud de Colombia, junto con las creencias culturales en torno al suicidio.

La percepción social comunitaria frente al fenómeno del suicidio es un tema que se ha estudiado por décadas. Fue Durkheim (1995) quien refirió que los hechos sociales influyen negativa o positivamente en la vida de las personas. Más específicamente Ramos (2006) señala sobre el suicidio que es un fenómeno social con connotaciones psicológicas.

De esta manera, los hechos relevantes de una sociedad tales como la corrupción, el desempleo, la violencia, las creencias religiosas, etc., los cuales fueron percibidos por los participantes, tal y como la manifiesta Durkheim (1995), pueden condicionar la voluntad de los individuos y también cambiar sus expectativas de vida y, entonces, puede surgir una acción represiva en sí mismo, que lo pone en predisposición desviando sus ideales a una causa desconocida que, de no tener cuidado, se puede convertir en un acto suicida (Ramos, 2006).

En cuanto a los factores protectores, existe diversidad de situaciones percibidas por los participantes que pueden prevenir el evento. Las categorías que surgieron de los grupos de discusión son casi las mismas que se derivaron en el estudio de los factores de riesgo. Los participantes reconocen que la familia es el principal factor protector contra el suicidio, puesto que es a partir de los valores inculcados, de la buena comunicación que pueda existir entre padres e hijos, de la comprensión y del amor incondicional. Romagnoli, Kuzmanic y Caris (2006) resaltan que la familia juega un papel sumamente importante en la prevención de conductas de riesgo en 
los niños y adolescentes. Resulta fundamental sensibilizar a los padres y/o adultos acerca de la importancia de su papel como agentes preventivos, promoviendo el desarrollo de factores protectores.

La disminución del riesgo del suicidio se hace con la formulación coordinada de estrategias y acciones que apunten al mejoramiento de las condiciones de salud y bienestar de las comunidades. Esta visión es compartida por Ojeda y Villalobos (2011), quienes enfatizan la importancia de la creación de políticas públicas con la participación de las comunidades en donde se estudien los factores de riesgo y lo más importante, se conozcan los factores protectores.

Finalmente, todo lo expuesto refleja la importancia de incluir a las comunidades en la construcción de políticas en pro de mejorar las condiciones de salud del municipio. La ventaja de este tipo de trabajos es que la construcción de nuevos conceptos y soluciones se genera a partir de las realidades de las comunidades quienes evidentemente comparten imaginarios e ideas sobre el tema que se pretendió analizar.

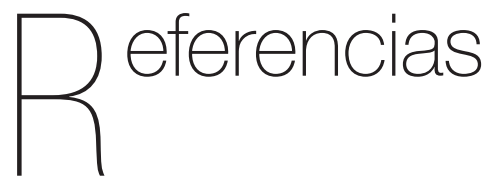

Álvarez, A., Ojeda, E., \& Martínez, N. (2010). Percepción social de la comunidad acerca del suicidio en pasto y Tumaco, Nariño: divergencias y puntos de encuentro. Universidad y salud, 10, 7-17.
Ansari, A. (2001). Risk factors associated with overdose among bahraini youth. Suicide and life-threatening behavior, 31, 197-206.

Arlaes, L., Hernadez, G., \& Alvares, C. (1998). Conducta suicida: factores de riesgo asociados. Universidad de nariño. Revista Cubana Medicina Integra, 14(2), 122-126

Blandón, A., Quintero, H., García, J., \& Layne, B. (2015). El suicidio: cuatro perspectivas. Medellín- Colombia: Fundación Universitaria Luis Amigó.

Blandón, O., \&Andrade, J. (2015). Aproximación al funcionamiento neurobiológico de la persona suicida. Medellin: Ediciones Funlam.

Bonilla, E., \& Rodríguez, P. (1997). Más allá del dilema de los métodos: las investigaciones sociales. Bogotá: Grupo Editorial Norma.

Borowsky, I. (2013). Suicidal thinking and behavior among youth involved in verbal and social bullying: risk and protective factors. Publimedical, 53(1), 4-12.

Bustamante, F., \& Florenzano, R (2013). Programas de prevención del suicidio adolescente en establecimientos escolares: una revisión de la literatura. Revista Chilena de Neuro-Psiquiatría, 51(2), 126-136.

Bronfenbrenner, U. (1977). La ecología del desarrollo humano. Barcelona: Paidós.

Cañón, S., Castaño. J., Atehortúa, B., Botero, P., García, L., Rodríguez, L., Tovar, C., \& Rincón, E. (2011). Factores de Riesgo Para Suicidio según dos cuestionarios y factores asociados en población estudiantil de la Universidad de Manizales. Psicología desde el Caribe, 29(3), 632-664. 
Durkheim, E. (1995). Un estudio en sociología. Nueva york.: Nueva York.

Hernández, R., Fernández, C., \& Baptista, P. (2010). Metodología de la investigación. México, D.F.: Mc Graw Hill.

Instituto Nacional De Medicina Legal (2012). Comportamiento suicida en Colombia. Bogotá. Recuperado el 7 de abril de 2015 de http://www.medicinalegal.gov.co/docu ments/10180/34861/6+5+suicidio+forens is+2012.pdf/654be518-60d0-4979-b899f26c83d1bb8c

Instituto Nacional de Medicina Legal (2013). Comportamiento suicida en Colombia. Bogotá. Recuperado el 7 de abril de 2015 de http://www.medicinalegal.gov.co/docu ments/10180/34861/6+5+suicidio+forens is+2013.pdf/654be518-60d0-4979-b899$\mathrm{f} 26 \mathrm{c} 83 \mathrm{~d} 1 \mathrm{bb} 8 \mathrm{c}$

Jiménez, K., Carballo, A., Cano. A., \& Marín, M. (2014). Relación entre el riesgo de suicidio, estrategias de afrontamiento y autoeficacia en dependientes del alcohol. Revista Salud y Drogas, 14(2), 121-129.

Keller, S., Sarchiapone, M., \& Zarrilli, F. (2011). Trkb gene expression and DNA methylation state in wernicke area does not associate with suicidal behavior. Journal of Affective Disorders, 135(1-3), 400-404.

Liam-Tam, P. (2011). Erception of suicidal attempts among college students in malaysia. Asian Social Science, 7(7) 30-41.

Mansilla, F. (2012). Factores presentes en la Conducta Suicida. Revista de ciencias sociales, 44, 24-29.
Mann, J. (2014). Surviving psychiatric illness: suicide risk assessment and prevention. Columbia University.

Matlyn, M., \& Foley, H. (1996). Sensación y percepción. México D. F.: Prentice Hall.

Martínez, A., Moracén, I., Madrigal, M., \& Almenares, M. (1998). Comportamiento de la conducta suicida infanto-juvenil. Revista Cubana Medicina Gen Integral, 14(6), 554-559

Monge, J., Cubillas, M., Román, R., \& Abril, E. (2007). Intentos de suicidio en adolescentes de educación media superior y su relación con la familia. Psicología y Salud, 17, 45-51.

Muñoz, E., \& Gutierrez, M. (2010). Factores de riesgo asociados al suicidio en Nariño. Revista Colombiana de Psiquiatría, 39(2), 291-312.

Ojeda, D., \& Villalobos-Galvis, F. (2011). Elementos para una política pública desde la comprensión del suicidio. Revista Colombiana de Psicología, 20(1), 57-73.

Organización Mundial de la Salud. (2002). Informe mundial sobre la violencia y la salud. Recuperado el 2015 de abril de 8, de http://www.who.int: http://www.who. int/violence_injury_prevention/violence/ world_report/es/summary_es.pd

Organización Mundial de la Salud. (2005). Comisión sobre determinantes sociales de la salud: hacia un marco conceptual que permita analizar los principales determinantes en salud. Ginebra.

Organización Mundial de la Salud. (2006). Informe regional de suicidio. Bogotá: Organización Panamericana de la Salud. 
Organización Mundial de la Salud. (2013). Salud mental: un estado de bienestar. Recuperado el 16 de abril de 2015 de http://www.who.int/features/factfiles/ mental_health/es/

Organización Mundial de la Salud. (2014). El suicidio, un problema de salud pública. Ginebra: Organización Mundial de la Salud.

Organización Mundial de la Salud. (2015). Suicidio y prevención. Recuperado el 2016 de enero de 18 de http://www.who.int/ mediacentre/factsheets/fs398/es/

Patton, M.Q. (1990). Qualitative evaluation and research methods (2nd ed.). New York: Newbury Park, CA.

Rocha, A. (2007). Calidad de Vida y Riesgo Suicida: Un Problema de Salud Pública. Revista Centro de Estudios en Salud, 1(8), 142-149.

Romero, M., \& Gonnet, J. (2013). Un diálogo entre Durkheim y Foucault a propósito del suicidio. Revista Mexicana de Sociología, 75(4), 589-616.

Sandoval, C. (1996). Programa de especialización en teoría, métodos y técnicas de investigación social, investigación cualitativa. Bogotá.: Arfo Editores e Impresores Ltda.
Sarmiento, Z., Sánchez, S., \& Vargas, Á. (2010). Conducta suicida y su relación con los factores de riesgo psicosociales. Medisan 14(8), 1054-1060.

Sistema Nacional de Vigilancia Epidemiológica. (2013). Sistema nacional de vigilancia epidemiológica reportes regionales. Recuperado el 2015 de abril de 18 de http://www.ins.gov.co/lineas-de-accion/ subdireccion-

Taha, N., Florenzano, R., Sieverson, C., Aspillaga, H., \& Alliende, L. (2011). La espiritualidad y religiosidad como factor protector en mujeres depresivas con riesgo suicida: consenso de expertos. Revista Chilena de Neuropsiquiatría, 49(4), 347-360.

Villalobos, F. (2007). Aplicación del modelo procesual del estrés a la conducta suicida. Universidad y salud, 8, 126-141.

Vianchá, M., Bahamon, M., \& Alarcón, L. (2013). Variables Psicosociales asociadas al Intento de Suicida, Ideación Suicida y Suicidio en Jóvenes. Tesis Psicológica, 8(1) 112-123. 